\title{
Effects of total knee arthroplasty on ankle alignment in patients with varus gonarthrosis: Do we sacrifice ankle to the knee?
}

\author{
Sarper Gursu ${ }^{1} \cdot$ Hakan Sofu $^{2} \cdot$ Peter Verdonk $^{3} \cdot$ Vedat Sahin $^{2}$
}

Received: 13 March 2015 / Accepted: 12 November 2015 / Published online: 21 November 2015

(C) European Society of Sports Traumatology, Knee Surgery, Arthroscopy (ESSKA) 2015

\begin{abstract}
Purpose Total knee arthroplasty is one of the most commonly preferred surgical methods in the treatment of patients with varus gonarthrosis. In this study, we aimed to evaluate the radiological changes observed in the ankles after total knee arthroplasty.

Methods Between May 2012 and June 2013, 80 knees of 78 patients with varus deformity over $10^{\circ}$ underwent total knee arthroplasty. For each patient, full-leg standing radiographs were obtained pre- and post-operatively. Mechanical and anatomical axes (HKA and AA), lateral distal femoral angle, medial proximal tibial angle, lateral distal tibial angle (LDTA), ankle joint line orientation angle (AJOA), tibial plafond talus angle (PTA) and talar shift were measured for each patient both pre- and post-operatively.

Results Pre-operatively, the mean HKA was $16.6^{\circ}$ and the mean AA was $10.41^{\circ}$, both in favour of varus alignment. Post-operatively, the mean HKA decreased to $3.6^{\circ}$ and the mean AA to -2.1 . The mean LDTA was $87.3^{\circ}$. Before the operation, the mean AJOA was $-7.6^{\circ}$, opening to the medial aspect of the ankle, and it was $0.04^{\circ}$ after the operation and opening to the lateral aspect $(p<0.05)$.

Conclusion Our study reveals the changes occurring in the ankle after acute correction of long-standing varus deformity of the knee using total knee arthroplasty. In cases
\end{abstract}

Sarper Gursu

sarper154@yahoo.com

1 Baltalimanı Bone and Joint Diseases Education and Research Hospital, Rumelihisari caddesi No: 62, Sariyer, 34470 Istanbul, Turkey

2 Erzincan University Faculty of Medicine, Erzincan, Turkey

3 Antwerp Orthopedic Center, Monica Hospitals, Antwerp, Belgium undergoing knee arthroplasty, effect of the acute change in the alignment of the knee on the ankle should be taken into consideration and the amount of correction should be calculated carefully in order not to damage the alignment of the ankle.

Level of evidence IV.

Keywords Varus gonarthrosis $\cdot$ Arthroplasty $\cdot$ Knee $\cdot$ Ankle $\cdot$ Alignment

\section{Introduction}

High-grade gonarthrosis is a global orthopaedic problem, affecting a rather big proportion of the elderly population $[2,18,24]$. Varus gonarthrosis occurs as a result of a longlasting process and, besides the knee neighbouring joints like the ankle and the hip, develops compensatory changes due to abnormal loading [9]. In patients with varus deformity in the lower extremities, attention is usually focused on the knees; however, the pathological findings as well as the other adaptational changes in the ankles and hips are usually neglected. According to Bellemans et al. [1], an important fraction of the normal population (32\% of men, $17 \%$ of women) has a natural alignment of $3^{\circ}$ or more varus, and the restoration of mechanical alignment to neutral in these cases may be unnatural for them. It has also been demonstrated that constitutional varus did not affect joint line orientation in the coronal plane [22].

Though there are numerous treatment options for highgrade knee osteoarthritis, total knee arthroplasty (TKA) is currently the most commonly preformed surgical modality $[3,6,8]$. Although it is known to be very successful not only in relieving pain in the knee but also in restoring the alignment of the lower limb, the effects of TKA on the 
ankles and the hips are not clear. It has been reported that disorders of the foot and ankle may be source of pain after TKA [14].

The main purpose of the present study was to evaluate the radiological changes observed in the ankle joint and its relation to subjective statement of ankle pain following acute correction of the alignment of the lower extremity using TKA for high-grade varus gonarthrosis.

\section{Materials and methods}

Between 2012 and 2013, 80 knees of 78 patients with a varus deformity over $10^{\circ}$ underwent TKA. Eighteen patients were males, and 60 were females. The median age of the patients was 67 (range 54-78) years. Forty-four patients were operated on the right side, 32 on the left side and 2 on both sides. The prosthetic design preferred was decided according to the individual conditions of each patient. For patients under 65 years, mobile-bearing TKA design was preferred, whilst for the ones over 65 years, fixed-bearing TKA design was preferred (Sigma Fixed
Bearing System, DePuy Orthopaedics Inc, Warsaw, IN, USA; NexGen LPS Flex Mobile, Zimmer Inc, Warsaw, IN, USA). The decision between the posterior cruciate ligament (PCL) retaining and substituting TKA was made intraoperatively according to the existence of the PCL. For each patient, full-leg standing radiographs were obtained both pre- and post-operatively. When obtaining radiographic images, patella and the ankle joints were marked on the skin for both legs of the patients to avoid rotational malpositioning. The distance between the two legs was standardized by the use of a standard sized radiolucent pad which was placed in between the medial femoral condyles, so that the post-operative changes observed in the ankles would be due to only the re-alignment of the extremity, achieved by TKA (Fig. 1). All full-leg standing radiographs were obtained digitally by DDR Inventor V (JSB Medics Co, Bucheon City, South Korea), and the measurements were taken by Infinitt PACS System (Infinitt Healthcare Co, Seoul, South Korea). Mechanical tibio-femoral angle (in other words hip-knee-ankle angle, HKA) and anatomical axes (AA), mechanical and anatomical lateral distal femoral angle (mLDFA and aLDFA), medial proximal
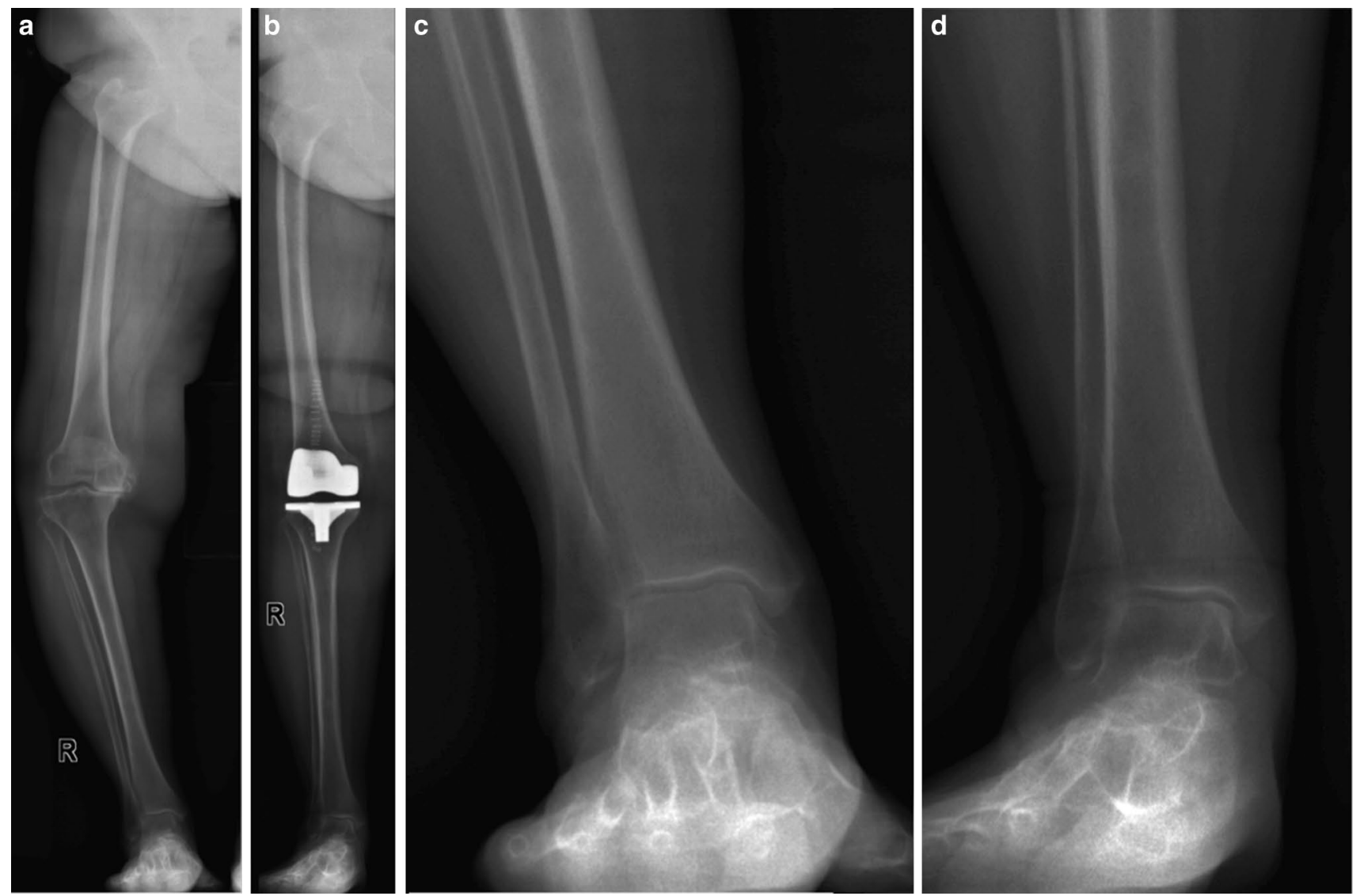

Fig. 1 a Pre-operative standing orthoroentgenograms of a patient with varus gonarthrosis. A radiolucent foam was placed in between the medial femoral condyles, $\mathbf{b}$ post-operative orthoroentgenogram of the same patient, $\mathbf{c}$ pre-operative alignment of the ankle, $\mathbf{d}$ the alignment of the ankle after total knee arthroplasty 
tibial angle (MPTA), lateral distal tibial angle (LDTA), ankle joint line orientation angle (AJOA), tibial plafond talus angle (PTA) and talar shift (TS) were measured for each patient both pre- and post-operatively (Fig. 2). Talar shift, which can also be expressed as medial clear space of the ankle joint, was described as the distance between joint surface of the medial malleolus and the medial joint surface of the talus on standard AP radiographic images. All measurements repeated separately for each patient were recorded by two of the observers. The interobserver reliability was 0.99 . For AJOA, a positive value was used to define an angle opening to the lateral aspect of the ankle and a negative value to define an angle opening to the medial aspect of the ankle. Similarly for PTA, a positive value meant an angle opening to the lateral aspect of the ankle and a negative value, an angle opening to the medial aspect. Clinically, subjective statement of ankle pain after TKA was also questioned and noted for all patients. Any relationship between ankle pain and AJOA was evaluated.

The procedures followed were in accordance with the ethical standards of the responsible committee on human experimentation and with the Helsinki Declaration of 1975, as revised in 2000. The current study has been approved by Erzincan University Faculty of Medicine Ethical

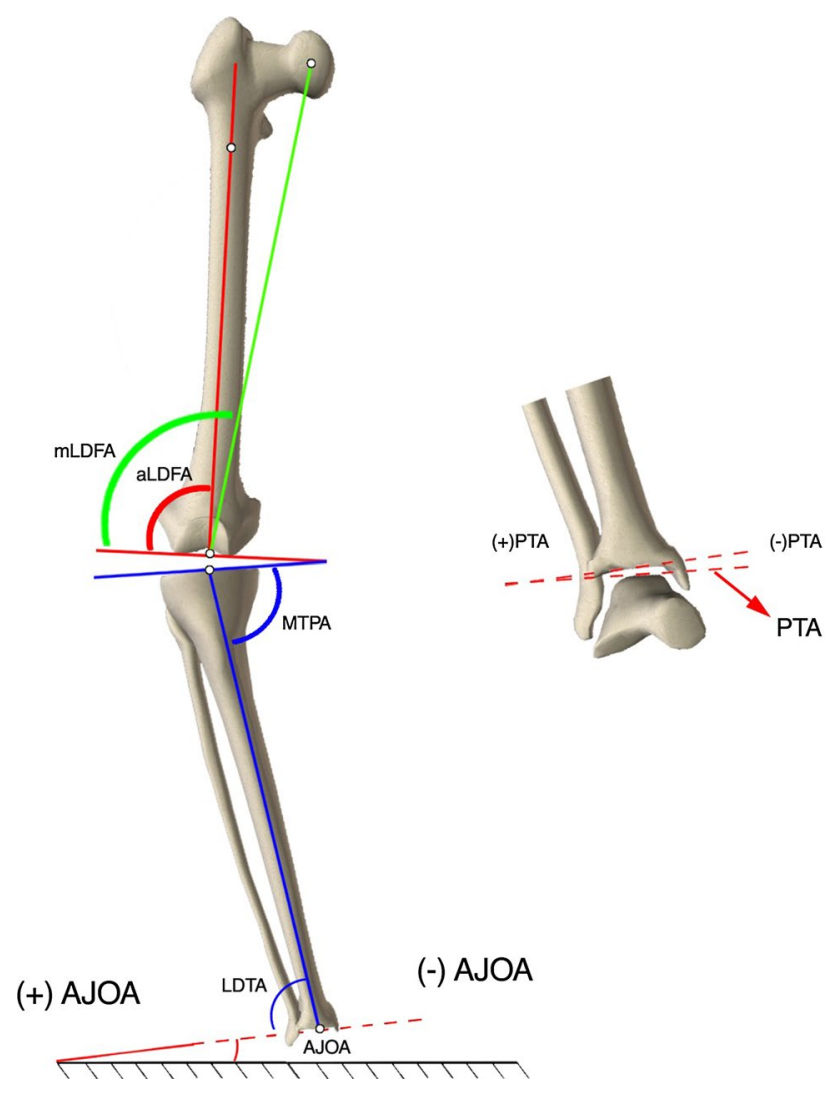

Fig. 2 Measurements taken on each orthoroentgenogram
Research Institutional Review Board (Document number: 44495147-050.02.04-E.36587).

\section{Statistical analysis}

All results were evaluated using SPSS 20.0 (IBM Corporation, New York, USA). The statistical evaluation of the difference between two dependent parameters was made using paired samples $t$ test, whilst the evaluation of two independent parameters was made by independent samples $t$ test. Dot plots, evaluating correlation between changes in HKA and AJOA, were performed. The level of statistical significance was set at $p \leq 0.05$.

\section{Results}

On the pre-operative full-leg standing radiographs, the mean HKA and the mean AA were both in favour of varus alignment. Post-operatively, the mean HKA decreased to $3.6^{\circ} \pm 3.6^{\circ}$ and the mean $\mathrm{AA}$ to $-2.1^{\circ} \pm 3.6^{\circ}$. Both decreases were significant $(p<0.05)$. The mean pre-operative mLDFA was $93.8^{\circ} \pm 2.2^{\circ}$, and the mean aLDFA was $87.6^{\circ} \pm 2.8^{\circ}$. The mean LDTA was $87.3^{\circ} \pm 6.9^{\circ}$, supporting lateral metaphyseal collapse, as a compensatory change. The mean MPTA increased significantly from pre-operative to post-operative period $(p<0.05)$. Pre-operatively, the mean amount of TS was measured as $2.8 \pm 0.8 \mathrm{~mm}$, whilst it was $3 \pm 0.9 \mathrm{~mm}$ post-operatively. Although the increase in the TS was significant, it was not for PTA (n.s.). Preoperatively, the mean angle between the ankle joint line and the ground line was $-7.5^{\circ} \pm 4.7^{\circ}$, opening to the medial aspect of the ankle; it was $0.04^{\circ} \pm 3.8^{\circ}$ post-operatively, opening to the lateral aspect of the ankle (Table 1) $(p<0.05)$.

For the patients with a varus deformity between $10^{\circ}$ and $15^{\circ}$, AJOA decreased to a mean of $0.4 \pm 3.9$ post-operatively from a mean of $-6.3^{\circ} \pm 4.3^{\circ}$ pre-operatively. The mean AJOA was $-8.4 \pm 7.8$ pre-operatively and measured as $-0.2 \pm 3$ post-operatively for the patients with a varus deformity over $15^{\circ}$. The changes in the two groups were both statistically significant $(p<0.05)$. Dot plots demonstrated a direct correlation between changes in HKA and AJOA from pre- to post-operative period (Fig. 3a, b). When the amount of change in PTA, TS and AJOA in patients with a varus deformity between $10^{\circ}$ and $15^{\circ}$ was compared to the patients with a varus deformity over $15^{\circ}$, there was no significant difference (n.s.) (Table 1).

Subjective statement of pain in ankle joint after TKA was noted in 19 of 80 cases. Thirteen of them had $>15^{\circ}$ preoperative varus deformity in the operated knee joint. The mean post-operative AJOA was $5^{\circ} \pm 1.9^{\circ}$ in those 13 cases, whereas it was $5.3^{\circ} \pm 1.3^{\circ}$ in the other 6 cases. Severity of 
Pre-operative

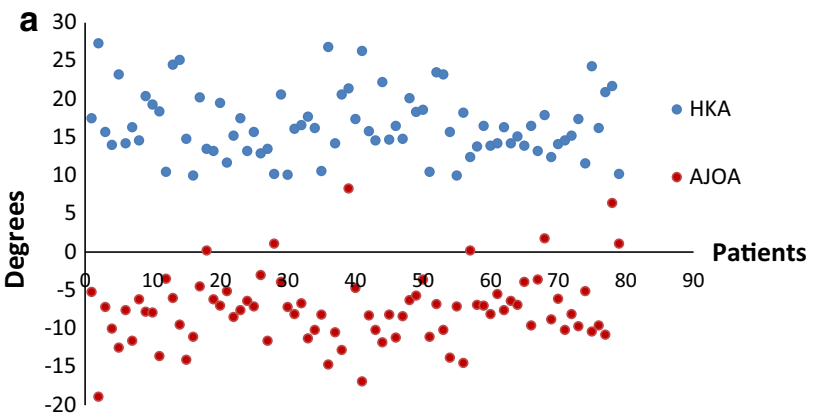

Post-operative

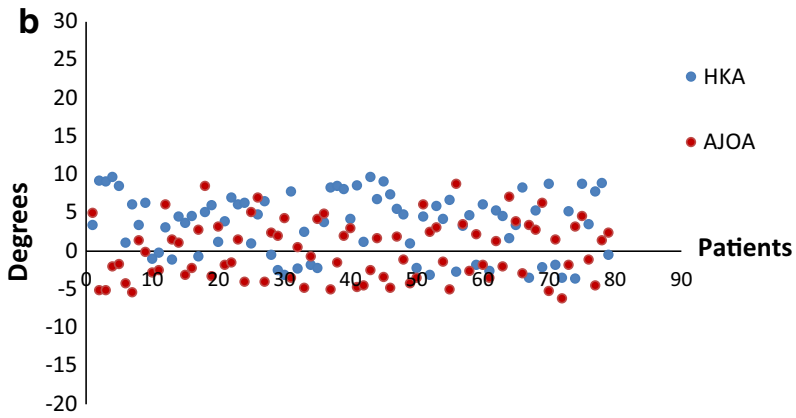

Fig. 3 a, b Dot plots demonstrating direct correlation between changes in HKA and AJOA from pre- to post-operative periods

the pre-operative varus deformity in the knee (varus $>15^{\circ}$ or between $10^{\circ}$ and $15^{\circ}$ ) was not significantly correlated with post-operative ankle pain (n.s.).

\section{Discussion}

The most important finding of the present study was that due to compensatory changes altering the orientation of the ankle in patients with long-standing severe varus gonarthrosis, acute correction in the alignment of the knee joint during TKA may lead to radiographic problems in the ankle joint which may potentially be related to secondary clinical complaints. Total knee arthroplasty is a very common surgical procedure used as the treatment method of patients with high-grade varus gonarthrosis [23]. One of the main aims of this surgical procedure is to restore the normal alignment of the lower extremity, with the attention being focused mostly around the knee joint $[4,10,13,17,21]$. Although TKA procedure is proven to be very successful in restoring the alignment of lower extremities, it certainly has some effects also on the neighbouring joints, the hips and the ankles, which are not that clear [9]. In the literature, there are only a few studies evaluating the effects of knee osteoarthritis on the ankles, but studies about the effects of TKA on the ankles are almost lacking $[7,15,16,19,20]$. 
Fig. 4 Achieving a valgus alignment in the knee could easily result in an overcorrection in the alignment of the ankle, and leaving a residual varus in the knee could give the best results
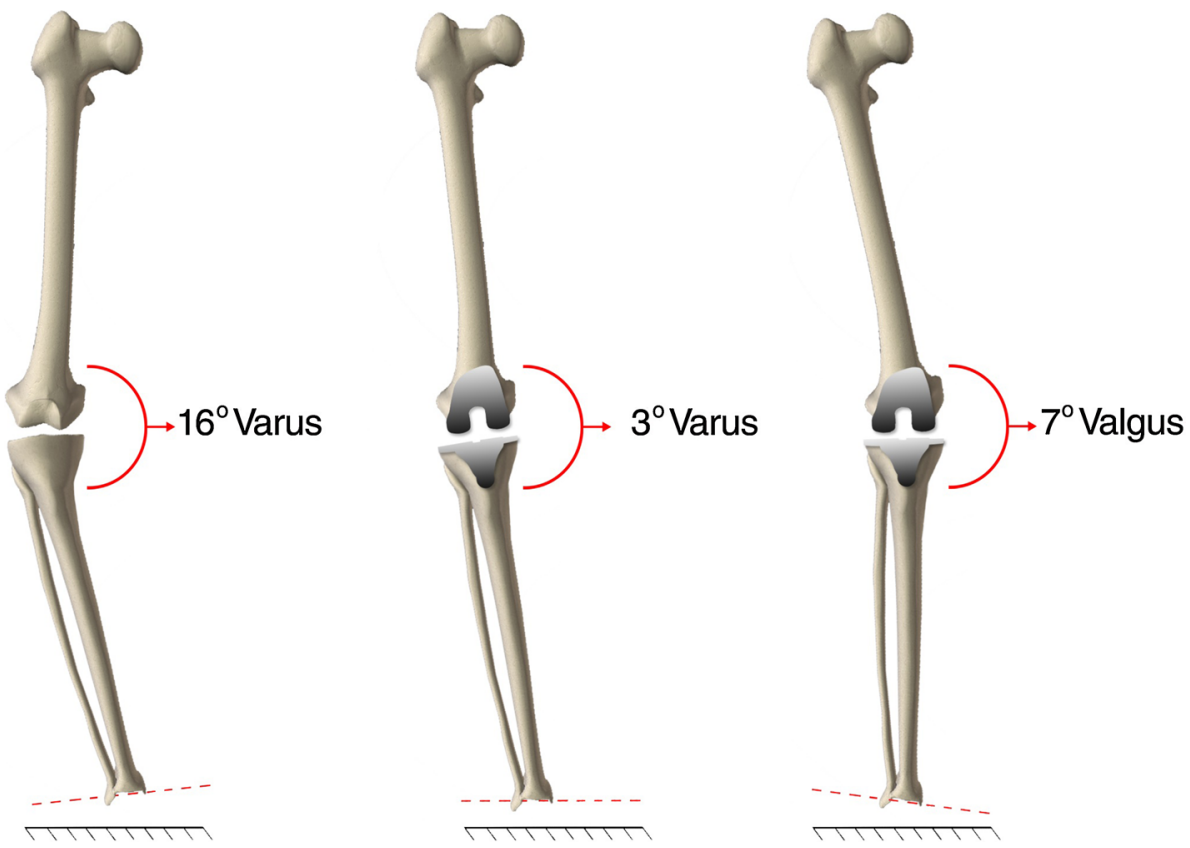

Issin et al. [9] evaluated 315 knees of 164 patients with varus gonarthrosis and concluded that abnormal forces applied to ankle caused collapse in the lateral distal tibial metaphysis. Tallroth et al.'s series included 104 patients operated due to knee osteoarthritis. They have evaluated the degenerative changes observed within the ankles and found that there was a significant correlation between the knee and ankle osteoarthritis [20]. In another study by Desai et al. [5], deformities of the foot were found to be effective on the alignment of the knees and the authors recommended including the foot deformity during preoperative evaluation and planning of knee deformities. Levinger et al.'s [12] study has revealed that medial compartment arthrosis in the knee joint was closely associated with a more pronated foot when compared to healthy controls. Another study demonstrating the relation between the structural changes in the knee and deviated ankle alignment was conducted by Hubbard et al. [7], and they praised the opinion about considering compensatory changes and altered mechanics of the ankle whilst addressing lower extremity functional deficits in patients with knee osteoarthritis. In our study, severe varus deformity was noted in all patients pre-operatively, and significant changes in their ankles according to radiographic measurements were observed after TKA. The decrease in LDTA and the failure of the parallelism between the ankle joint line and the ground line were the most obvious changes in the ankles.

A thorough review of the literature revealed that Lee and Jeong held the unique study about the effects of TKA on degenerative changes observed in the ankle. In their study, the authors reported that arthritis has developed or progressed in many of their cases, with either a varus or a valgus deformity, after a mean of 3 years. Their conclusion was that in case of a larger varus, the arthritic changes occurred much significantly and earlier [11]. In the current study, all patients had varus deformity over $10^{\circ}$ and they were treated with TKA. Pre-operatively, the mean ankle joint orientation line was significantly varus with respect to the ground line and the LDTA was less than $90^{\circ}$ possibly due to abnormal loading in the ankle. Post-operatively, the ankle joint orientation line was almost parallel to the ground line and the improvement was significant. There was no significant change in the mean PTA. Although the change in the mean TS was significant, it was only $0.2 \mathrm{~mm}$.

The mean pre-operative varus deformity was so severe that the optimal valgus alignment could not be achieved in most of the cases, and a mean residual varus deformity was valid. Still, the improvement in the alignment of the ankle was significant regardless of the amount of preoperative varus deformity in the knees as the joint line was parallel to the ground in most of the cases. As the patients had long-standing varus in their knees, there were compensatory changes in the ankles such as the decrease in the LDTA. Thus, we conclude achieving a valgus alignment in patients with long-standing varus deformity in their knees could easily lead to an overcorrection in the alignment of the ankle and leaving a residual varus in the knee could be considered in order to prevent the ankle from malalignment (Fig. 4). This could also prevent the ankle from early degeneration and arthritic changes reported in Lee and Jeong's study [11].

There are some limitations of the present study. First, the cohort was not large but is of reasonable size. Second, the patients were not followed long enough to evaluate whether 
these changes in the ankle lead to osteoarthritis or not. On the other hand, the present study may be a reference for long-term clinical trials and also future investigations to provide a better understanding of mechanical changes affecting the ankle joint after TKA and their importance to cause secondary clinical problems. We recommend that in patients undergoing TKA, effect of the acute change in the alignment of the knee on the ankle joint should be taken into consideration in order not to damage the stability and the mechanics of the ankle.

\section{Conclusion}

During TKA in patients with severe long-standing varus gonarthrosis, the effect of the acute correction in the alignment of the knee on the ankle should be taken into consideration. A neutral alignment or a minimal varus could be the optimal position of the legs in order not to lead to malalignment and potential clinical complaints in the ankle. Further clinical studies with larger cohorts and long-term follow-up are required for a definitive answer.

Acknowledgments There was no external source of funding for this study.

\section{Compliance with ethical standards}

Conflict of interest The authors declare that they have no conflict of interest.

\section{References}

1. Bellemans J, Colyn W, Vandenneucker H, Victor J (2012) Is neutral mechanical alignment normal for all patients: the concept of constitutional varus. Clin Orthop Relat Res 470(1):45-53

2. Blazek K, Favre J, Asay J, Erhart-Hledik J, Andriacchi T (2013) Age and obesity alter the relationship between femoral articular cartilage thickness and ambulatory loads in individuals without osteoarthritis. J Orthop Res 32(3):394-402

3. Chen JY, Yeo SJ, Yew AK, Tay DK, Chia SL, Lo NN, Chin PL (2014) The radiological outcomes of patient-specific instrumentation versus conventional total knee arthroplasty. Knee Surg Sports Traumatol Arthrosc 22(3):630-635

4. Daniilidis K, Tibesku CO (2013) Frontal plane alignment after total knee arthroplasty using patient-specific instruments. Int Orthop 37(1):45-50

5. Desai SS, Shetty GM, Song HR, Lee SH, Kim TY, Hur CY (2007) Effect of foot deformity on conventional mechanical axis deviation and ground mechanical axis deviation during single leg stance and two leg stance in genu varum. Knee 14(6):452-457

6. Harding P, Holland AE, Delany C, Hinman RS (2013) Do activity levels increase after total hip and knee arthroplasty? Clin Orthop Relat Res 472(5):1502-1511

7. Hubbard TJ, Hicks-Little C, Cordova M (2010) Changes in ankle mechanical stability in those with knee osteoarthritis. Arch Phys Med Rehabil 91(1):73-77
8. Iacono F, Bruni D, Bignozzi S, Colle F, Marcacci M (2014) Does total knee arthroplasty modify flexion axis of the knee? Knee Surg Sports Traumatol Arthrosc 22(8):1728-1735

9. Issin A, Sahin V, Koçkara N, Gürsu S, Kurtuldu A, Yildirim T (2012) Is proximal tibia the major problem in varus gonarthrosis? Evaluation of femur and ankle. Eklem Hastalik Cerrahisi 23(3): $128-133$

10. Keyes BJ, Markel DC, Meneghini RM (2013) Evaluation of limb alignment, component positioning, and function in primary total knee arthroplasty using a pinless navigation technique compared with conventional methods. J Knee Surg 26(2):127-132

11. Lee JH, Jeong BO (2012) Radiologic changes of ankle joint after total knee arthroplasty. Foot Ankle Int 33(12):1087-1092

12. Levinger P, Menz HB, Fotoohabadi MR, Feller JA, Bartlett JR, Bergman NR (2010) Foot posture in people with medial compartment knee osteoarthritis. J Foot Ankle Res 3:29

13. Luyckx T, Vanhoorebeeck F, Bellemans J (2015) Should we aim at undercorrection when doing a total knee arthroplasty? Knee Surg Sports Traumatol Arthrosc 23(6):1706-1712

14. Manning BT, Lewis N, Tzeng TH, Saleh JK, Potty AG, Dennis DA, Mihalko WM, Goodman SB, Saleh KJ (2015) Diagnosis and management of extra-articular causes of pain after total knee arthroplasty. Instr Course Lect 64:381-388

15. Muehleman C, Margulis A, Bae WC, Masuda K (2010) Relationship between knee and ankle degeneration in a population of organ donors. BMC Med 8:48

16. Onodera T, Majima T, Iwasaki N, Kamishima T, Kasahara Y, Minami A (2012) Long-term stress distribution patterns of the ankle joint in varus knee alignment assessed by computed tomography osteoabsorptiometry. Int Orthop 36(9):1871-1876

17. Paternostre F, Schwab PE, Thienpont E (2014) The difference between weight-bearing and non-weight-bearing alignment in patient-specific instrumentation planning. Knee Surg Sports Traumatol Arthrosc 22(3):674-679

18. Prieto-Alhambra D, Judge A, Javaid MK, Cooper C, Diez-Perez A, Arden NK (2014) Incidence and risk factors for clinically diagnosed knee, hip and hand osteoarthritis: influences of age, gender and osteoarthritis affecting other joints. Ann Rheum Dis 73(9): 1659-1664

19. Reilly K, Barker K, Shamley D, Newman M, Oskrochi GR, Sandall S (2009) The role of foot and ankle assessment of patients with lower limb osteoarthritis. Physiotherapy 95(3):164-169

20. Tallroth K, Harilainen A, Kerttula L, Sayed R (2008) Ankle osteoarthritis is associated with knee osteoarthritis. Conclusions based on mechanical axis radiographs. Arch Orthop Trauma Surg 128(6):555-560

21. Thienpont E, Paternostre F, Pietsch M, Hafez M, Howell S (2013) Total knee arthroplasty with patient-specific instruments improves function and restores limb alignment in patients with extra-articular deformity. Knee 20(6):407-411

22. Victor JM, Bassens D, Bellemans J, Gürsu S, Dhollander AA, Verdonk PC (2014) Constitutional varus does not affect joint line orientation in the coronal plane. Clin Orthop Relat Res 472(1):98-104

23. Yan CH, Chiu KY, Ng FY (2011) Total knee arthroplasty for primary knee osteoarthritis: changing pattern over the past 10 years. Hong Kong Med J 17(1):20-25

24. Zhang J, Song L, Liu G, Zhang A, Dong H, Liu Z, Li X, Luo J (2013) Risk factors for and prevalence of knee osteoarthritis in the rural areas of Shanxi Province, North China: a COPCORD study. Rheumatol Int 33(11):2783-2788 